\title{
Tribology of Confined Fomblin-Z Perfluoropolyalkyl Ethers: Role of Chain-End Chemical Functionality
}

\author{
Marina Ruths*,† and Steve Granick* \\ Department of Materials Science and Engineering, University of Illinois, Urbana, Illinois 61801
}

Received: April 20, 1999; In Final Form: August 6, 1999

\begin{abstract}
Strong dependence of the tribological response upon the nature of the polar end groups of perfluorinated linear chains was observed for Fomblin-Z perfluoropolyalkyl ethers of similar length and composition but terminated by a different polar group at both chain ends. The number-average molecular weight of the polymers was $M_{\mathrm{n}} \approx 2000 \mathrm{~g} \mathrm{~mol}^{-1}$ and the chain-end functionality was either carboxylic acid, hydroxyl, piperonyl, or the $p$-phenoxyanilinium salt of a carboxylic acid. The method of investigation was a surface forces apparatus modified for dynamic oscillatory shear at variable frequency and effective shear rate. Differences were observed as concerns not only the shear forces but also the minimum thickness to which the films could be compressed under a given normal load and the adhesion measured on separation of the surfaces after prior compression. The shear forces were studied at normal pressures of 1 and $3 \mathrm{MPa}$, both in the linear viscoelastic regime and at high shear amplitudes corresponding to shear rates of $10^{-2}-10^{5} \mathrm{~s}^{-1}$. The carboxylic acid terminated polymer displayed solidlike responses to shear, possibly reflecting dimerization owing to hydrogen bonding. This contrasted with the more fluidlike shear rheology of the hydroxyl- and piperonyl-terminated polymers, in which the association from hydrogen bonding and polar interactions is believed to be weaker and result in a different structure. The sample comprised of the $p$-phenoxyanilinium salt of a carboxylic acid could not be compressed to less than an exceptionally large film thickness, around $100 \AA$, and did not appear to solidify at the pressures studied. This study suggests that not only the affinity of the functionalized chain ends to a solid surface but also the self-association of polar end groups in the nonpolar environment of fluorinated chains influences the lubricating performance of these films.
\end{abstract}

\section{Introduction}

Studies of the friction of molecularly thin fluid films confined between atomically smooth solid surfaces have till present largely concentrated on molecules chosen to be uniform in their chemical composition. Especially as concerns polymer melts, a survey of the literature shows that homopolymer systems were selected (for example, polysiloxanes, ${ }^{1-4}$ polybutadiene, ${ }^{1,5}$ or perfluorinated perfluoropolyethers ${ }^{6-8}$ ) in which the chain end was of essentially the same composition as the polymer backbone. In these systems, much of the shear response can be attributed to bridging of some of the chains from one surface to another. ${ }^{9-12}$ However, we may expect to see a different response if the ends of linear chains carry a polar functionality. (1) The strength of bridging may be enhanced if two ends of a chain become adsorbed to opposed surfaces. (2) The strength of bridging may be diminished if the two chain-ends are so strongly adsorbed that they cannot cross from one surface to another. (3) Self-association of polar end groups, in a nonpolar environment of repeat segments along the polymer chain, may become a prominent effect.

To examine these possibilities, we have studied perfluorinated polymers of similar molecular weight but different chain-end functionality. We are interested here in perfluoropolyalkyl ethers (PFPAE) of the so-called Fomblin-Z series, which are linear, random copolymers of perfluoroethylene oxide and perfluoromethylene oxide. ${ }^{13,14}$ These fluids possess excellent thermal

\footnotetext{
$\dagger$ Present address: Max-Planck-Institut für Polymerforschung, Ackermannweg 10, D-55128 Mainz, Germany.
}

10.1021/jp991278k CCC: $\$ 18.00$ and chemical stability, low vapor pressure, and favorable lubricating properties. They are currently used as lubricants in aerospace $^{15-17}$ and laboratory ultrahigh vacuum equipment ${ }^{18}$ and in magnetic storage devices (computer disk drives). ${ }^{19-40}$ There is considerable interest in further improvement of the lubricating properties of Fomblin-Z polymers and of their ability to protect sliding surfaces against wear. Several end-functionalized Fomblin-Z polymers are widely used in commercial magnetic storage devices. ${ }^{19-36,38-40}$ This family of chain polymers therefore provides a useful system in which to examine the influence of chain-end functionality.

We have studied the static and dynamic (shear) interactions between opposed atomically smooth surfaces separated by molecularly thin films of four different difunctionalized Fomblin-Z oligomers with a molecular weight around $2000 \mathrm{~g} \mathrm{~mol}^{-1}$. The end groups studied were carboxylic acid, hydroxyl, and piperonyl (3,4-(methylene dioxy)benzyl) groups and a $p$ phenoxyanilinium salt of a carboxylic acid. Two of these samples, the hydroxyl- and piperonyl-terminated chains, are currently being widely used commercially for lubrication of computer hard disks. ${ }^{19-36,38-40}$ It has been found experimentally that the introduction of carboxyl, hydroxyl, or piperonyl end groups increases the ability of the Fomblin-Z polymer to protect the underlying substrate against wear. ${ }^{19-29}$ It has been demonstrated that these polar end groups are preferentially located near the polar surface of the hydrogenated carbon overcoat that forms the top layer of the computer hard disk ${ }^{22,30-32,39,40}$ and also that they are located near other polar surfaces such as silica ${ }^{21,23,33,34,41}$ and $\mathrm{ZrO}_{2} \cdot{ }^{42}$ Heating ("thermal bonding") introduces chemical 
TABLE 1: Properties of Difunctionalized Fomblin-Z with the General Structure $\mathrm{E}-\mathrm{CF}_{2} \mathrm{O}-\left(\mathrm{CF}_{2} \mathrm{CF}_{2} \mathrm{O}\right)_{m}-\left(\mathrm{CF}_{2} \mathrm{O}\right)_{n}-\mathrm{CF}_{2}-\mathrm{E}$

\begin{tabular}{lllccc}
\hline \multicolumn{1}{c}{ sample } & \multicolumn{1}{c}{ end groups, $-\mathrm{E}$} & $m / n^{a}$ & functionality $^{a}$ & $M_{\mathrm{n}}{ }^{a}$ & viscosity $^{b}(\mathrm{P})$ \\
\hline DIAC & $-\mathrm{COOH}$ & 0.87 & 0.95 & 2100 & $2.01(290 \mathrm{~K}), 0.657(313 \mathrm{~K})$ \\
DOL & $-\mathrm{CH}_{2} \mathrm{OH}$ & 1.12 & 0.96 & 2100 & $1.85(290 \mathrm{~K}), 0.733(313 \mathrm{~K})$ \\
AM2001 & $-\mathrm{CH}_{2} \mathrm{OCH}_{2} \mathrm{C}_{6} \mathrm{H}_{3} \mathrm{OOCH}_{2}$ & 1.00 & 0.96 & 2300 & $1.03(299 \mathrm{~K}), 0.53(314 \mathrm{~K})$ \\
$p$-phenoxyanilinium salt & $-\mathrm{COO}^{-} \mathrm{H}_{3} \mathrm{~N}^{+} \mathrm{C}_{6} \mathrm{H}_{4} \mathrm{OC}_{6} \mathrm{H}_{5}$ & 0.87 & 0.95 & 2300 & $27.71(317 \mathrm{~K}), 8.14(343 \mathrm{~K})$
\end{tabular}

of carboxylic acid

${ }^{a}$ The samples were characterized at Hitachi Corp., Japan, by means of NMR. ${ }^{b}$ The samples were characterized at Hitachi Corp., Japan, by means of capillary viscometry (Ubbelohde viscometer).

bonds between functionalized chain ends and polar groups on the surface to further improve the stability of the lubricant film. ${ }^{19,21-25,28,29,39,40}$

For the low molecular weight polymers (2000-4000 $\mathrm{g} \mathrm{mol}^{-1}$ ) currently utilized in magnetic storage devices, the polar end groups constitute a large fraction of the whole sample and affect strongly or even dominate the bulk properties of the sample, a phenomenon called the "copolymer effect". ${ }^{43,44}$ In the study described below, we demonstrate that not only the affinity of the end groups for a polar surface but also the different structures that can be inferred for confined films containing different polar moieties in a nonpolar environment are critical for the performance of the film as a lubricant.

Our experimental method gives information on the forces acting across the film as the surfaces are brought toward and from each other in a carefully controlled manner, and thus on the film thickness formed under different compressive and tensile pressures. Dynamic shear measurements at different normal pressures over a wide range of shear rates $\left(10^{-2}-10^{5}\right.$ $\mathrm{s}^{-1}$ ) gave information on the rheological properties of confined films of thickness comparable to the desired thickness of lubricant on a computer hard disk for "contact" recording (tens of angstroms). ${ }^{19,29,38}$ With this technique it was also possible to observe surface deformations (caused by trapping of liquid or even plastic deformation of substrates) and damage of the solid surfaces, in case such effects occurred. The substrate used was muscovite mica, which allowed us to study shearing of the film between atomically smooth surfaces in a contact that can be thought of as one single asperity. Thermal bonding is, however, not feasible on mica surfaces since they do not expose functional groups.

The results will be compared to shear experiments on high molecular weight unfunctionalized Fomblin- $Z^{6,7}$ and branched, unfunctionalized Fomblin- $\mathrm{Y}^{6}$ and to linear and branched unfunctionalized perfluoropolyalkyl ethers of different backbone structure $^{8}$ but similar molecular weight as the samples in this study.

\section{Materials and Methods}

Perfluoropolyalkyl Ether Samples. The carboxylic acid (DIAC), hydroxyl- (DOL), and piperonyl-functionalized (AM2001) Fomblin- $Z^{45}$ were obtained from Ausimont. The $p$ phenoxyanilinium-terminated sample was synthesized at Hitachi Corp., Japan, by ion exchange of the carboxylic acid terminated Fomblin-Z. The structures of the Fomblin-Z backbone and the functional end groups are shown in Table 1 together with some parameters describing each sample. The polydispersities (ratio of weight-average to number-average molecular weight, $M_{\mathrm{w}} /$ $M_{\mathrm{n}}$ ) are around 1.1-1.2. ${ }^{14,46}$ Higher molecular weight Fomblin-Z samples, including unfunctionalized polymer, were also available and were the topic of a separate investigation. ${ }^{47}$ However, unfunctionalized Fomblin- $Z$ of a molecular weight similar to the one of the functionalized oligomers in this study, $2000 \mathrm{~g}$ $\mathrm{mol}^{-1}$, was not commercially available.
The samples in this study are all transparent liquids at room temperature. DIAC and DOL were colorless, AM2001 was slightly yellow, and the $p$-phenoxyanilinium salt was redbrown. It was readily apparent from its sluggish flow properties in the sample container that the $p$-phenoxyanilinium salt had a much higher bulk viscosity than the other samples (cf. Table 1).

To remove possible dust particles and humidity, approximately $1 \mathrm{~mL}$ of the DIAC and DOL samples were dissolved in approximately $10 \mathrm{~mL}$ of a hydrofluoroether fluid, HFE-7100 $\left(\mathrm{C}_{4} \mathrm{~F}_{9} \mathrm{OCH}_{3}\right.$, nonafluoro-1-methoxy butane, a product of $3 \mathrm{M}$ Corp.) and kept over molecular sieves (Aldrich, pore size $5 \AA$ ) overnight. The solutions were then filtered through a MillexSR syringe filter (Millipore, pore size $0.5 \mu \mathrm{m}$ ) and the solvent evaporated under vacuum at room temperature for $48 \mathrm{~h}$. This solvent has been developed as a replacement for chlorofluorocarbons and does not decompose to form compounds that contribute to the depletion of the ozone layer. ${ }^{48}$ It was found that solutions of AM2001 in HFE-7100 caused discoloration of the molecular sieves. The use of sieves was thus avoided for this sample, which was only dissolved in HFE-7100, filtered, and dried under vacuum as described above.

Unlike the three samples discussed above, the $p$-phenoxyanilinium salt was only sparsely soluble in HFE-7100 and also in perfluoroheptane. A portion of this sample was dried under vacuum without preceding dissolution and filtration. It was found that it could be dissolved in a 1:1 mixture of ethanol and methanol, which was used to clean the instrument after use with this sample. One experiment on DIAC and one on AM2001 were also performed on portions of the samples that had only been dried under vacuum without dissolving and filtration. Some dust particles were found to be present in these samples, but the interactions and shear properties measured on particle-free contact positions were identical to the ones obtained for the samples treated as described above. The dried samples were kept in dry atmosphere in a desiccator when not in use.

The glassware used was cleaned overnight in a mixture of concentrated $\mathrm{H}_{2} \mathrm{SO}_{4}$ and Nochromix, rinsed with distilled water, kept in $2 \mathrm{M} \mathrm{KOH}$ overnight, rinsed again copiously in distilled water, and finally dried in an oven. It was difficult to completely remove these polymers from glass since their solubility in other liquids than perfluorinated solvents is generally low and the adsorbed layer to some extent protects a glass surface against etching agents. To ensure that no contamination was introduced, new glassware was used for each sample and discarded after the dissolving, drying, and filtering steps described above.

Measurements of Static Interactions. The investigations of film thickness, interaction forces, and thin-film shear rheology were performed using a surface forces apparatus (SFA) built in our laboratory, modeled after the "Mark II" SFA 49 and equipped with a device for oscillatory lateral shear..$^{3,50,51}$ All experiments were performed at $25^{\circ} \mathrm{C}$. The surfaces used were thin sheets of muscovite mica, which were silvered on one side and glued with the silvered side down onto fused silica disks with a half- 
cylindrical surface. The separation between the mica surfaces was measured by multiple beam interferometry. ${ }^{52}$ The distance resolution and thus the accuracy of the measurements of film thickness was $1-2 \AA$.

The conceivable solubility in the functionalized Fomblin-Z of the substances used to attach mica sheets onto the supporting silica disks was investigated. The gluing of the mica sheets involves initial melting of the glue substance by placing it on the heated silica disk, then placing a mica sheet onto the melted glue droplet such that capillary action causes the mica sheet to conform to the curvature of the underlying substrate, and finally solidification of the glue as the temperature is lowered by removal of the assembly from the hot plate. The candidate glues were either a 1:1 mixture of galactose and dextrose or 1,5diphenylcarbazide. Both have convenient melting temperatures and both are commonly used to glue mica sheets for SFA work. To study possible solubility, droplets of the different Fomblin-Z samples were placed in glass Petri dishes and a small amount of the sugar mixture or of diphenylcarbazide was added to each drop. The mixtures were kept at room temperature and examined under an optical microscope at different times for up to 1 week. Diphenylcarbazide crystals were readily soluble in DIAC, since the polymer directly in contact with the crystals turned bright purple already after less than $1 \mathrm{~h}$, and were also slightly soluble in the $p$-phenoxyanilinium salt but did not appear to be soluble in the hydroxyl- or piperonyl-terminated polymer.

The mixture of sugar crystals did not appear to dissolve in DIAC or DOL. Only after 1 week of contact between the sugar and the polymer was a very slight solubility in AM2001 detected, and similarly for the $p$-phenoxyanilinium salt, in which the solubility was even smaller.

It was thus found that the sugar mixture was the better choice of glue for this study. During the experiments, care was taken to use mica pieces that were large enough to completely cover and extend outside the edge of the supporting silica disks in order to minimize contact between the sample and the glue. The experiments were started approximately $30 \mathrm{~min}$ after inserting the polymer drop and finished within 3-4 h. The samples were not left in contact with the supports for longer times.

It should be noted that plastic deformation of the sugar glue may take place at large normal pressures; care had to be taken to ensure that an applied pressure did not cause a permanent flattening of the surfaces. This problem has been discussed in detail in ref 53.

During the experiment, the experimental chamber was kept dry by exposing the atmosphere to a reservoir of $\mathrm{P}_{2} \mathrm{O}_{5}$. After calibration of the contact thickness between the mica surfaces in dry air (separation distance $D=0$ ), a drop of dried polymer was inserted between the separated surfaces with a clean and dry glass pipet. The first three samples in Table 1 were slightly viscous, which might cause time effects during approach and separation of the surfaces (compression of the polymer film).$^{54}$ The $p$-phenoxyanilinium-terminated sample was extremely viscous, which made measurements particularly difficult. To facilitate the measurements in these systems, the stiffness of the force-measuring spring was chosen to be $930 \mathrm{~N} / \mathrm{m}$, which is an order of magnitude stiffer than commonly used for force measurements on simple liquids. Consequently, the sensitivity of the force measurements presented, around $10^{-7} \mathrm{~N}$, is an order of magnitude less than usually quoted for the SFA, ${ }^{55}$ but still sufficient for accurate determination of the forces expected in our systems.
During approach and subsequent retraction of the opposed surfaces, the waiting time between displacing the base of the spring and subsequently measuring the surface separation was varied. For relatively large film thickness (>1000 ̊), forces were measured approximately $1 \mathrm{~min}$ after a typical displacement of ca. $150 \AA$. For lesser film thickness, the distance change per step was decreased somewhat and the waiting time was increased successively so that the points where $F / R \approx 0$ in parts $\mathrm{A}-\mathrm{C}$ of Figure 1 were measured at waiting times of a few minutes, the points at the beginning of the repulsive regime (100-120 $\AA$ from the hard-wall) with waiting times of $10-15$ min and the points close to and on the "hard-wall" at approximately $20 \mathrm{~min}$ intervals (also on separation). The movement of the surfaces was found to have stopped completely during the times chosen. For the $p$-phenoxyanilinium-terminated sample (Figure 1D), longer waiting times were used, 20-30 min per data point. The waiting times will be discussed further below.

At points where the change in force with distance, $\mathrm{d} F / \mathrm{d} D$, is larger than the spring constant, there will be an instability and a spontaneous jump to the next stable region. Such jumps were seen upon separation of the surfaces from an adhesive minimum in the force curves in Figure 1A-D.

Measurements of Shear Interactions. The dynamic measurements where performed with a device described in detail elsewhere $3,50,51$ that allows one to apply dynamic oscillatory shear forces over a wide range of frequencies and to measure the resistance to shear motion of the confined thin film. The device, on which the upper surface in the surface forces apparatus is mounted, consists of a rigid surface holder (boat) supported by two piezoelectric bimorphs attached to leaf springs, one on each side of the boat. For the device used in this study, the spring constant of this assembly was $1.6 \times 10^{4} \mathrm{~N} / \mathrm{m}$ (measured from its resonance frequency). To operate this device, a sinusoidal voltage is applied to one of the bimorphs, which causes it to deflect and the upper surface to move back and forth horizontally over the lower surface (oscillatory shear). The amplitude and phase of the displacement of the upper surface (with the surfaces in contact or separated, with or without sample) is detected at the input frequency with the other bimorph by means of a sensitive lock-in amplifier. When the surfaces are coupled (in adhesive contact or separated by a thin film), the movement of the upper surface is reduced and the resulting deflection of the detecting bimorph (and thus the output voltage) is then lower than the values obtained with the surfaces separated. The elastic (in-phase) and viscous (out-of-phase) compliance of the device itself (mainly arising from the glue) were determined for different input parameters from the difference in amplitude and phase with the mica surfaces in adhesive contact and separated. This predominantly elastic contribution can be separated from the total response when a sample is present between the surfaces according to the procedure described in ref 3 so that the elastic and viscous response of the confined film under study is obtained as a function of input frequency, displacement amplitude, film thickness (i.e., resulting strain or shear rate), and normal pressure.

The experiments in this study were performed in a frequency range of $0.13-130 \mathrm{~Hz}$ and with a shear displacement amplitude of $0.2-2000 \AA$. The pressure normal to the surfaces was changed with the distance controls described above. The methods we employed have been used to study a large variety of systems, from simple liquids to linear and branched polymers confined between mica or hydrocarbon surfaces. ${ }^{2-4,56}$ It is 

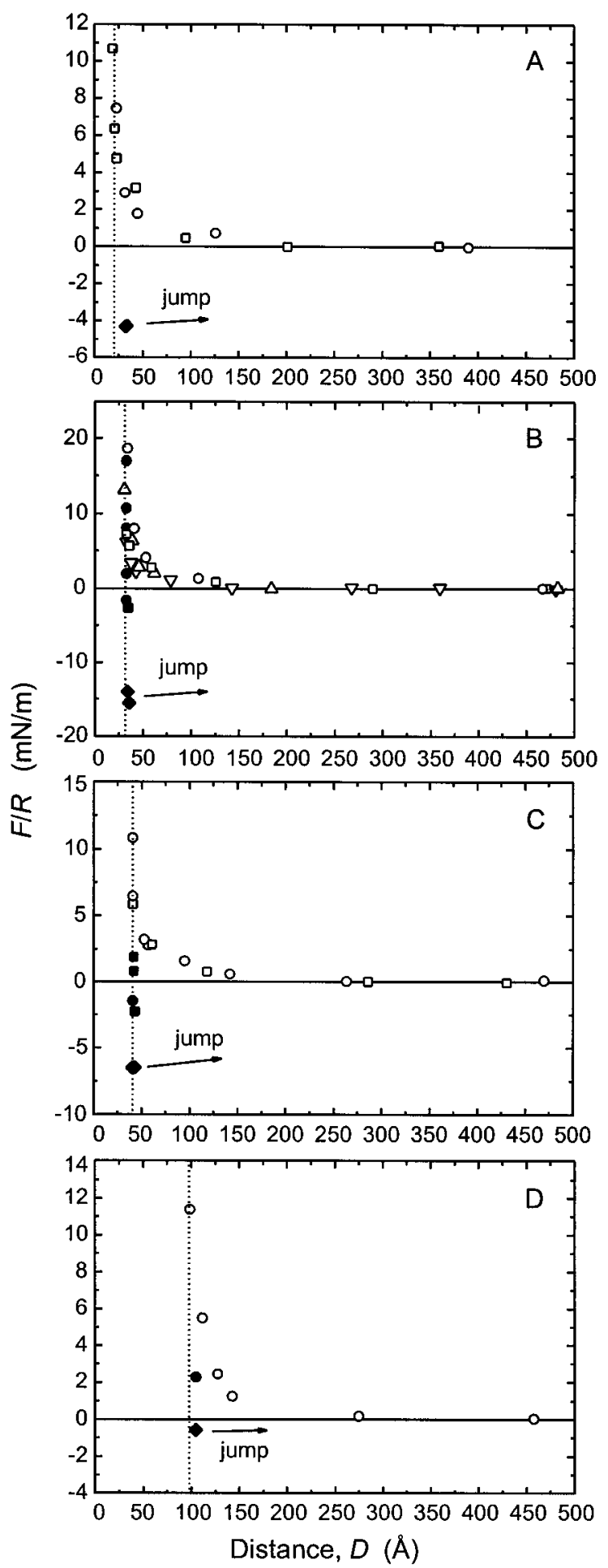

Figure 1. The static force, $F$, normalized by the mean radius of curvature, $R$, is plotted against the thickness of the liquid film between two solid surfaces, $D$, for Fomblin-Z perfluoropolyalkyl ethers of similar molecular weight $\left(M_{\mathrm{n}} \approx 2000 \mathrm{~g} \mathrm{~mol}^{-1}\right)$ but different chain-end functionality: (A) carboxylic acid, DIAC; (B) hydroxyl, DOL; (C) piperonyl, AM2001; (D) p-phenoxyanilinium salt of carboxylic acid. The value $D=0$ is the thickness of two opposed mica surfaces in adhesive contact in dry air atmosphere. Open symbols denote values measured upon approach and filled symbols denote values measured upon separation of the surfaces. The points of spontaneous jump apart upon separation are indicated by diamonds. The final thickness obtained on large compression of the film ("hard-wall repulsion") is indicated by the dotted vertical lines. This thickness was 24,33 , 40, and $97 \AA$ in panels $\mathrm{A}, \mathrm{B}, \mathrm{C}$, and $\mathrm{D}$, respectively. In Figures 1-7, squares and circles indicate results from independent experiments on different pairs of mica surfaces. generally observed that the properties of thin films go from liquidlike at large film thicknesses and low pressures toward more solidlike at higher confinement. ${ }^{2-4,56}$

\section{Results}

Force-Distance Measurements. Static interaction forces (normalized by the mean radius of curvature of the undeformed solid surfaces, $R$ ) are plotted against film thickness in Figure 1. Care was taken to equilibrate these measurements, as described in the Experimental section. For all four samples, a monotonic repulsion and a final, incompressible layer thickness ("hard-wall repulsion") were observed on approach. In each case, the range of the monotonic repulsion appeared at a separation of 100-120 $\AA$ from the final hard-wall separation indicated by the dotted vertical lines in Figure $1 \mathrm{~A}-\mathrm{D}$. On separation of the surfaces, adhesion of different strength for different samples was observed.

The hard wall thickness for each sample (cf. Figure 1A-D) was $24,33,40$, and $97 \AA$, respectively. On separation, the confined layers were found to expand to a thickness of 34,36 , 42, and $105 \AA$ before the jump apart.

Linear Regime of Oscillatory Shear. The response of the confined films to small-amplitude oscillatory shear at relatively low normal pressure, $1 \mathrm{MPa}$, is presented on log-log scales in Figure 2 as the elastic and viscous moduli vs angular frequency $(\omega=2 \pi f, f=0.13-130 \mathrm{~Hz})$. The shear amplitude was a few angstroms, small enough to maintain linear viscoelastic response. At this pressure, the film thicknesses, which are specified in the Figure legend, were only slightly larger than the "hard-wall" thickness for each sample given above. It is known that the pressure across the contact zone is not strictly constant when curved solid surfaces are compressed against one another; it may vary in a Hertzian ${ }^{57}$ (parabolic) manner or in a more complex fashion if the opposed surfaces adhere ${ }^{58}$ at the same time that they are compressed. In the comparisons made here, we have normalized the applied normal force by the measured contact area to obtain the mean normal pressure. Similarly, we have normalized the shear forces by the measured contact area to obtain the shear stresses.

At this pressure, $1 \mathrm{MPa}$, the DIAC sample showed a markedly different behavior from the three other samples. The elastic component dominated the shear response of the DIAC at all frequencies studied, which is an indication that these frequencies exceeded the inverse longest shear relaxation time of the confined polymer. The response of this sample was thus rubberlike, which has been observed for other confined linear polymers in this frequency regime..$^{3,4}$

The magnitudes of the moduli for the other three samples (Figure 1B-D) were considerably lower. For DOL and the $p$-phenoxyanilinium-terminated sample, a regime of predominantly elastic response at low frequencies was followed by a mainly viscous response at larger frequencies. The AM2001 showed a mainly viscous response at the lowest frequencies (i.e., a liquidlike response), followed by an elastic, and finally, at the largest frequencies studied, a regime of viscous response. The magnitudes of the moduli for this sample were between the results obtained for DOL and the $p$-phenoxyanilinium salt.

The transition from linear to nonlinear viscoelastic response as the input shear amplitude was increased is shown in Figure 3 , where the elastic and viscous moduli of the films are shown as functions of the strain (deformation amplitude divided by film thickness) at a frequency of $f=1.3 \mathrm{~Hz}$ for DIAC, DOL, and AM2001, and at $f=0.52 \mathrm{~Hz}$ for the $p$-phenoxyanilinium salt. The values of film thickness were the same as in Figure 2. 


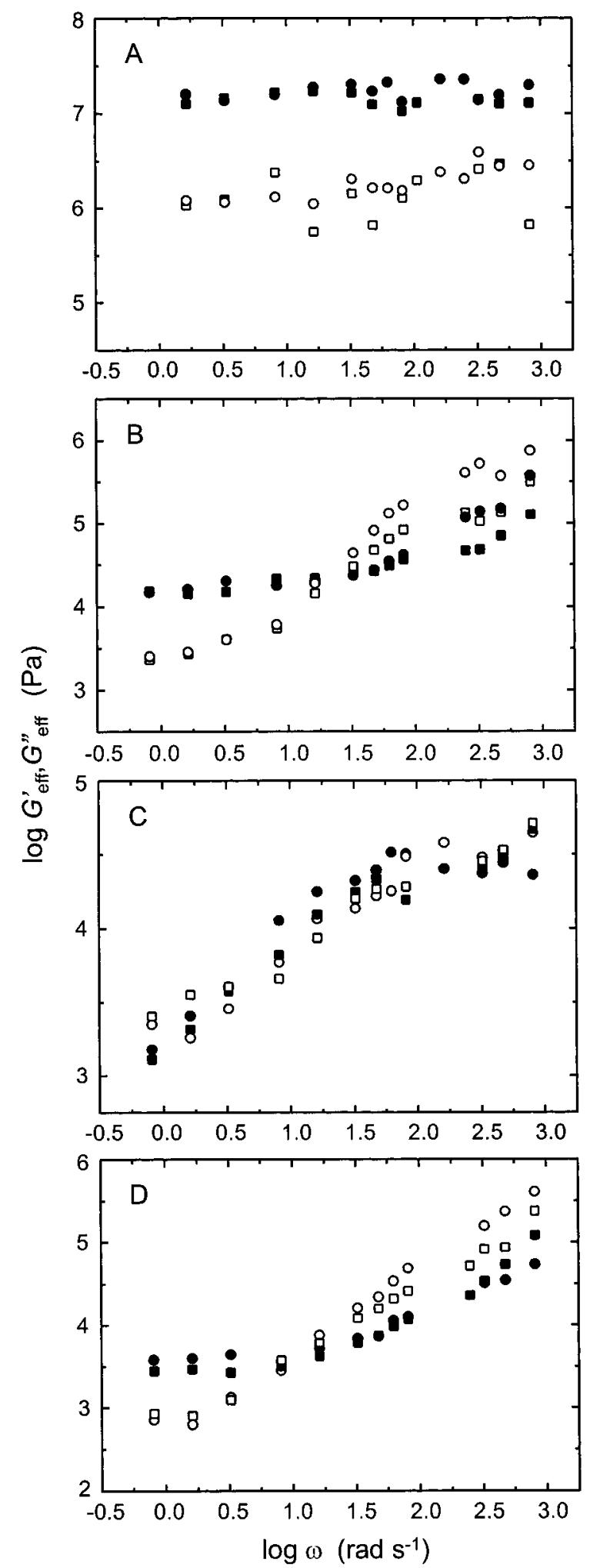

Figure 2. The effective storage modulus ( $G_{\text {eff }}^{\prime}$, filled symbols) and loss modulus ( $G^{\prime \prime}$ eff, open symbols), measured in the regime of linear viscoelastic response, are plotted on $\log -\log$ scales as a function of the angular frequency of oscillatory shear, $\omega$, for the case of relatively low pressure in the normal direction, $1 \mathrm{MPa}$. The film thickness, $D$, for the different samples was: (A) carboxylic acid (DIAC), $D=25$ $\AA$ A; (B) hydroxyl (DOL), $D=34 \AA$; (C) piperonyl (AM2001), $D=42$ $\AA$; (D) $p$-phenoxyanilinium salt of carboxylic acid, $D=98 \AA$.

At low deformation amplitudes, all samples showed a linear viscoelastic response. The transition from linear to nonlinear response occurred at the lowest strain $(\gamma)$ for DIAC $(\gamma=0.05$ at $f=1.3 \mathrm{~Hz}$ and $\gamma=0.1$ at $130 \mathrm{~Hz}$ ) and at the highest strain,
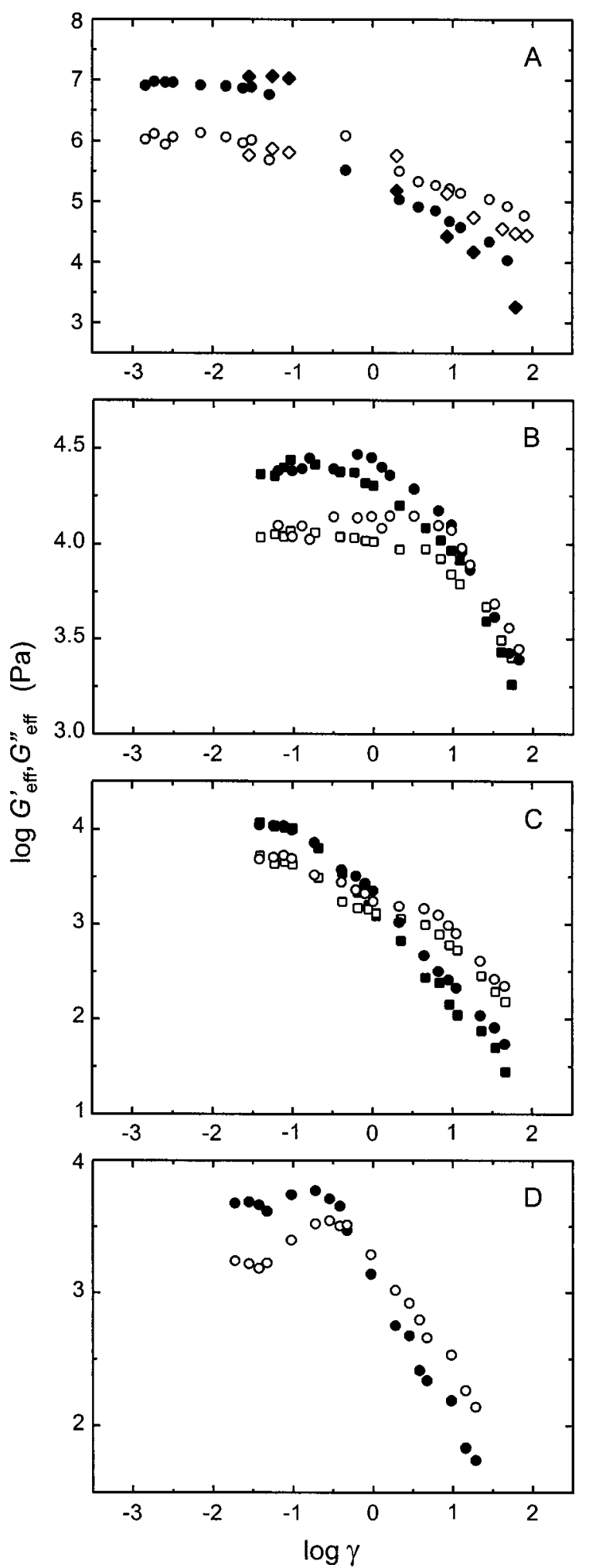

Figure 3. The effective storage modulus ( $G_{\text {eff }}^{\prime}$, filled symbols) and loss modulus ( $G^{\prime \prime}{ }_{\text {eff }}$, open symbols) are plotted as a function of the effective strain (deformation amplitude divided by film thickness) as the amplitude of the oscillatory shear deformation was increased. The experiments were performed at low pressure, $1 \mathrm{MPa}$, at a frequency of $1.3 \mathrm{~Hz}$ for (A) carboxylic acid, DIAC; (B) hydroxyl, DOL; and (C) piperonyl, AM2001 and at $0.52 \mathrm{~Hz}$ for (D) $p$-phenoxyanilinium salt of carboxylic acid. The diamond symbols in panel A show results from an additional experiment at $130 \mathrm{~Hz}$. The values of film thickness were the same as for Figure 2. A discontinuous transition, which is indicative of stick-slip sliding, was observed for DIAC but not for the other samples, where the transition from linear to nonlinear response was continuous and indicated smooth sliding at this normal pressure. 
$\gamma \approx 3$, for DOL. At this low pressure, the transition was discontinuous for the DIAC sample (indicating that that the commencement of macroscopic-sized sliding in this system occurred as a slip) and continuous for the other three samples. The decrease in moduli after the transition can be described as shear thinning.

In Figure 4, the viscous responses from Figure 3 are presented as the shear stress (force divided by area) as a function of the effective shear rate (strain multiplied by angular frequency). In the regime of linear response, the stress increased linearly with shear rate as expected for a Newtonian response. In the nonlinear regime, the shear stress increased much more slowly with shear rate.

Seldom in bulk systems is it possible to achieve shear rates sufficiently large that the shear stress fails to continue to increase with further increase of the shear rate (a few examples ${ }^{59-63}$ are known). A plateau of shear stress, a so-called limiting shear stress, is common to observe in lubricated systems, however. ${ }^{64-67}$ We have also observed this phenomenon here. In Figure 4, one observes that the limiting value of the shear stress, at the transition from linear to nonlinear response, was clearly different between the different samples, thus depending on the nature of the chain end group.

Responses at Increased Pressure. The results presented above (Figures 2-4) can be compared to the response to oscillatory shear of DOL, AM2001, and the $p$-phenoxyanilinium salt at identical input parameters but at a higher load or pressure, $3 \mathrm{MPa}$, which is shown in Figures 5-7. (Due to its unfavorable lubricating properties already at low pressure, the DIAC was not investigated at higher pressure). The normal pressure has been defined as discussed in the previous section. The values of film thickness at this pressure were the hard-wall thicknesses shown with dotted lines in Figure 1. The increase in pressure caused a shift in the shear response toward more elastic (rubberlike) response in all samples, although a regime dominated by viscous response could still be detected at the lowest frequencies in the AM2001 system (Figure 5B). As the shear amplitude at $f=1.3 \mathrm{~Hz}$ was increased (Figure 6), we observed a distinctly discontinuous transition from linear to nonlinear response for DOL at a strain of 0.07 (at $130 \mathrm{~Hz}$ the transition occurred at a slightly larger strain, $\gamma=0.13$ ) and a less obvious discontinuous transition for the AM2001 sample at $\gamma=0.1$. For the $p$-phenoxyanilinium salt film, the transition was continuous also at this pressure. In Figure 7, the viscous response shown in Figure 6 is presented as shear stress vs shear rate. For DOL, the value of the limiting stress was very similar to the value obtained at low pressure, while for the other samples, the limiting shear stress increased with increased normal pressure.

\section{Discussion}

Forces Acting upon Compression of the Films. As the thickness of the confined film was decreased slowly, a repulsive force was measured starting at 100-120 $\AA$ from the final hardwall separation (Figure 1). In systems comprised of small linear or spherical molecules, the interaction force has been found to oscillate as a function of separation distance. ${ }^{55}$ Such a layered structure has also been found in poly(dimethylsiloxane) films thinner than $50 \AA .68$ The range of the repulsive force measured for the functionalized Fomblin- $Z$ (Figure 1) is, however, too large to be the envelope of an oscillatory force caused by layering, which will be discussed further below.

It is known that the viscosity of polymer melts affects the force measurements if the equilibration time between the displacement of the base of the spring and the subsequent
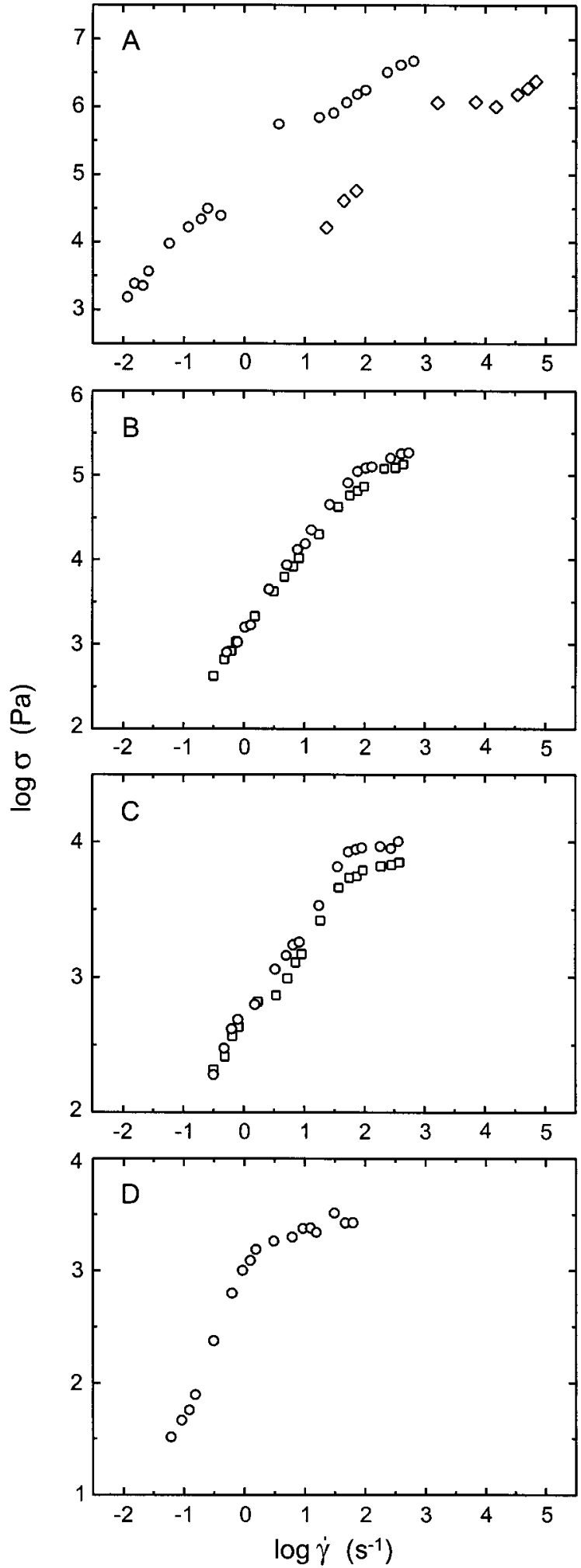

Figure 4. The data for the effective loss modulus shown in Figure 3 are plotted as viscous shear stress $(\sigma)$ vs shear rate (strain multiplied by angular frequency). As in Figure 3, the diamond symbols in panel A show results from an additional experiment conducted at $130 \mathrm{~Hz}$. A plateau stress level ("limiting shear stress") was reached at strains (cf. Figure 3 ) of approximately $0.05-0.1,3,2$, and 0.3 for (A) carboxylic acid, DIAC; (B) hydroxyl, DOL; (C) piperonyl, AM2001; and (D) $p$-phenoxyanilinium salt of carboxylic acid, respectively.

measurement of separation distance is too short. This "viscous drag" has been calculated by Horn et al. ${ }^{54}$ for systems of different bulk viscosity. For our spring stiffness of $930 \mathrm{~N} / \mathrm{m}$, radius of curvature $R=1.2-1.8 \mathrm{~cm}$, and viscosity of ap- 

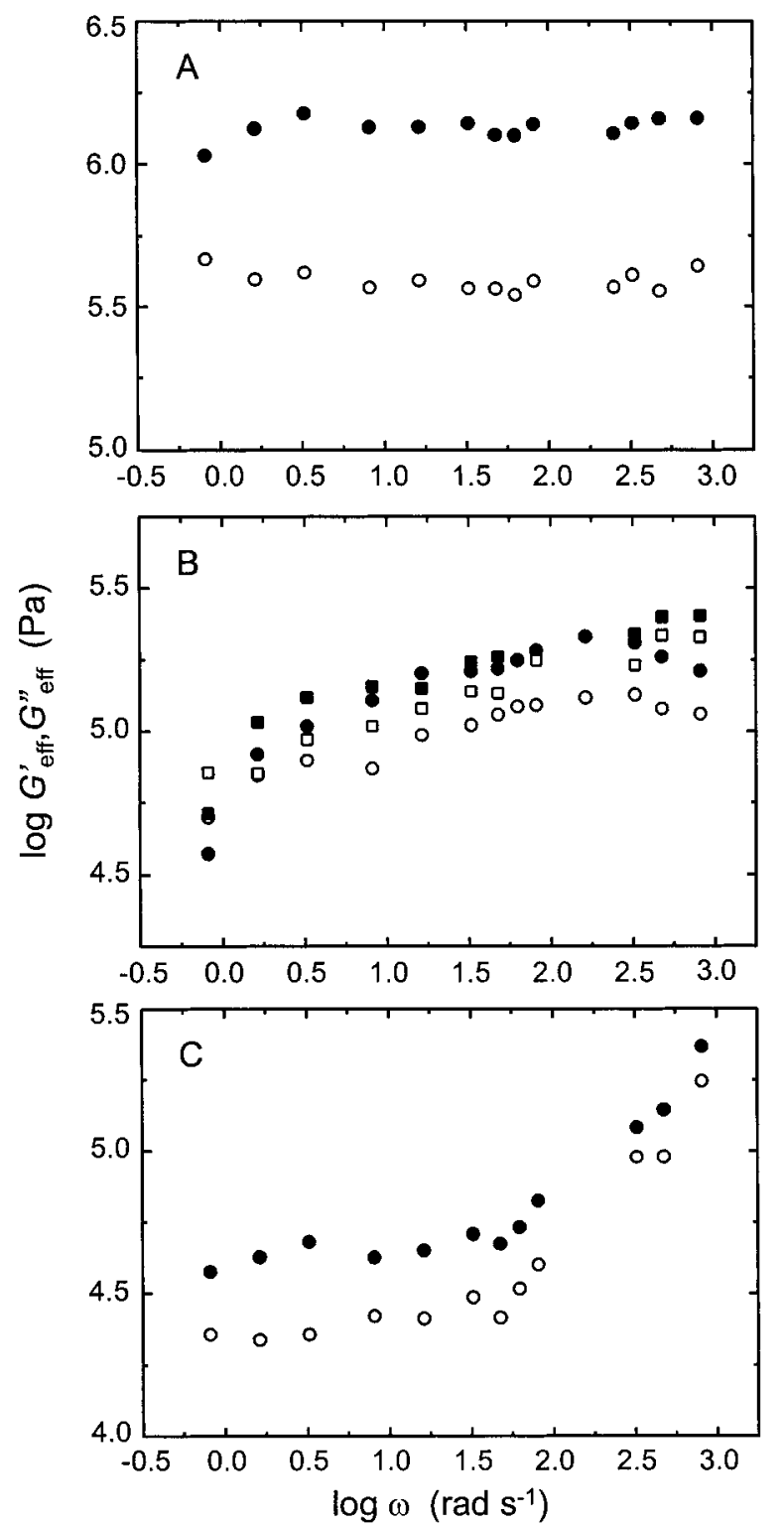

Figure 5. The effective storage modulus ( $G_{\text {eff }}^{\prime}$, filled symbols) and loss modulus ( $G^{\prime \prime}$ eff, open symbols), measured in the regime of linear viscoelastic response, are plotted on $\log -\log$ scales as a function of the angular frequency of oscillatory shear, $\omega$, at higher normal pressure than in the previous figures, $3 \mathrm{MPa}$. The film thickness measured at this pressure was the same as the final film thickness for each sample indicated in Figure 1. The panels denote (A) hydroxyl (DOL), $D=33$ $\AA$; (B) piperonyl (AM2001), $D=40 \AA$; and (C) $p$-phenoxyanilinium salt of carboxylic acid, $D=97 \AA$.

proximately $1.5 \mathrm{P}$ (estimated for $25^{\circ} \mathrm{C}$ from the data in Table 1) for the samples in Figure $1 \mathrm{~A}-\mathrm{C}$, the equilibration time after an inward step of $100 \AA$ should be equal to or less than 6 min (depending on the radius) at a separation of $100 \AA$ from the "hard-wall" (immobilized surface layer) and $20 \mathrm{~min}$ at $20 \AA$ from the hard-wall. ${ }^{54}$ The equilibration times chosen (cf. Experimental section) for these three polymers should thus be sufficient to avoid effects from viscous drag. For the extremely viscous $p$-phenoxyanilinium-terminated sample (Figure 1D), the calculation according to ref 54 suggests that the equilibration times already at larger separations would be on the order of hours. Interestingly, such long times were not necessary in order to observe experimentally that the surfaces had come to a steadystate position for this system, and the range of the repulsion was not longer than for the three less viscous samples. This
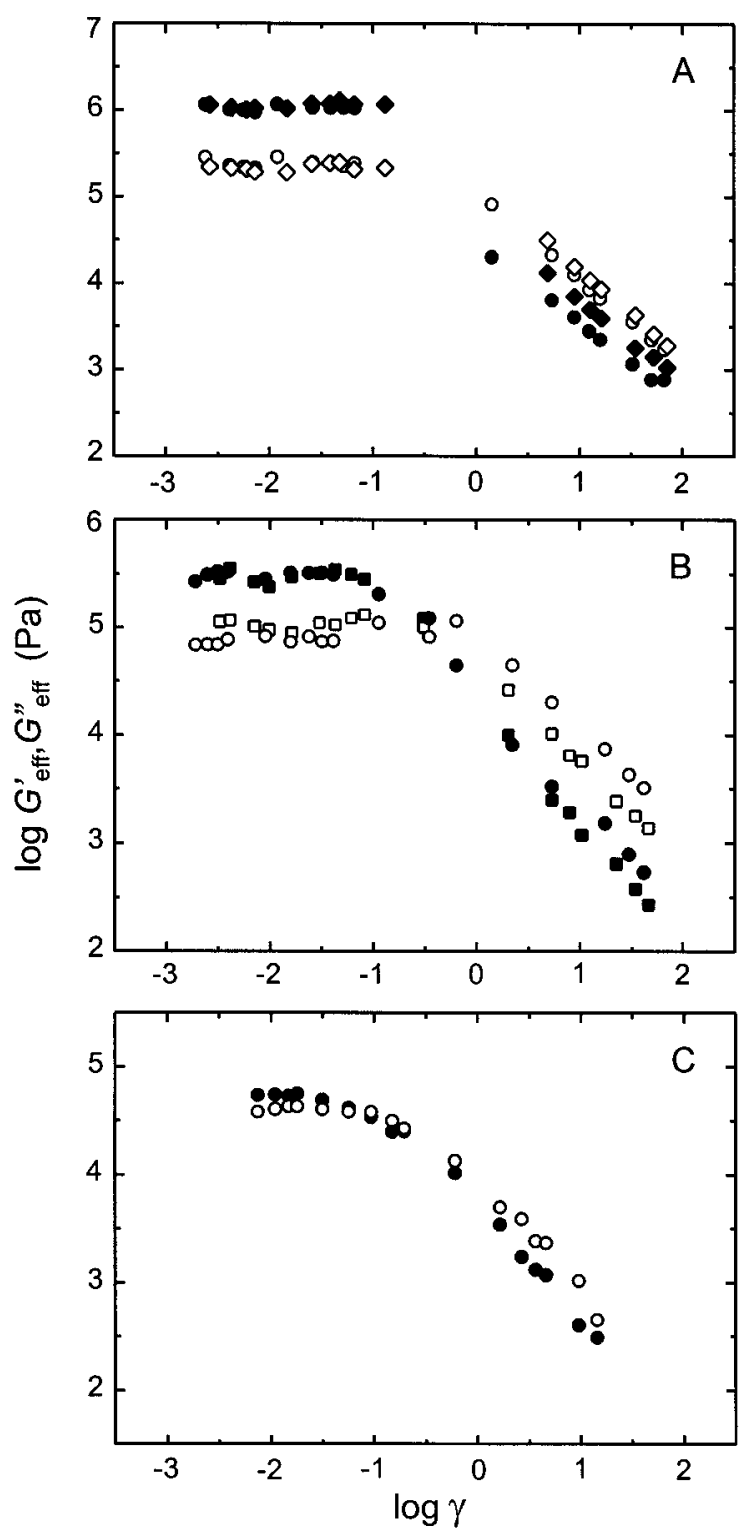

Figure 6. The effective storage modulus ( $G_{\text {eff }}^{\prime}$, filled symbols) and loss modulus ( $G^{\prime \prime}$ eff, open symbols) are plotted as a function of the effective strain, at a normal pressure of $3 \mathrm{MPa}$, as the amplitude of the oscillatory shear was increased. The experiments for (A) hydroxyl, DOL; (B) piperonyl, AM2001; and (C) p-phenoxyanilinium salt of carboxylic acid were all performed at $1.3 \mathrm{~Hz}$. The diamond symbols in panel A show results from an additional experiment at $130 \mathrm{~Hz}$. The film thicknesses were the same as for Figure 5. A distinct discontinuous transition from smooth to stick-slip sliding was observed for DOL and a smaller discontinuous transition for AM2001. For the $p$ phenoxyanilinium salt, the motion was continuous regardless of strain amplitude.

illustrates the difficulties in predicting thin-film flow behavior based on viscosity expected for the bulk state.

Force measurements have been performed in a similar manner on several unfunctionalized Fomblin-Z samples: a sample of higher molecular weight $\left(M_{\mathrm{n}}=2900 \mathrm{~g} \mathrm{~mol}^{-1}\right)$ with viscosity of $0.52 \mathrm{P}$ at $25^{\circ} \mathrm{C} ; 4^{47}$ a sample with a molecular weight around $10000 \mathrm{~g} \mathrm{~mol}^{-1}$ (Y.-K. Cho, University of Illinois, unpublished results) and a sample of $M_{\mathrm{n}}=12800 \mathrm{~g} \mathrm{~mol}^{-1}$. ${ }^{6}$ The repulsion in these samples appeared at a smaller distance, approximately $50 \AA$, from the hard-wall separation obtained for each sample. It has been noted for other polymer systems that it is generally difficult to reach the equilibrium situation and that the shape of the repulsive curve is similar to the interaction between end- 

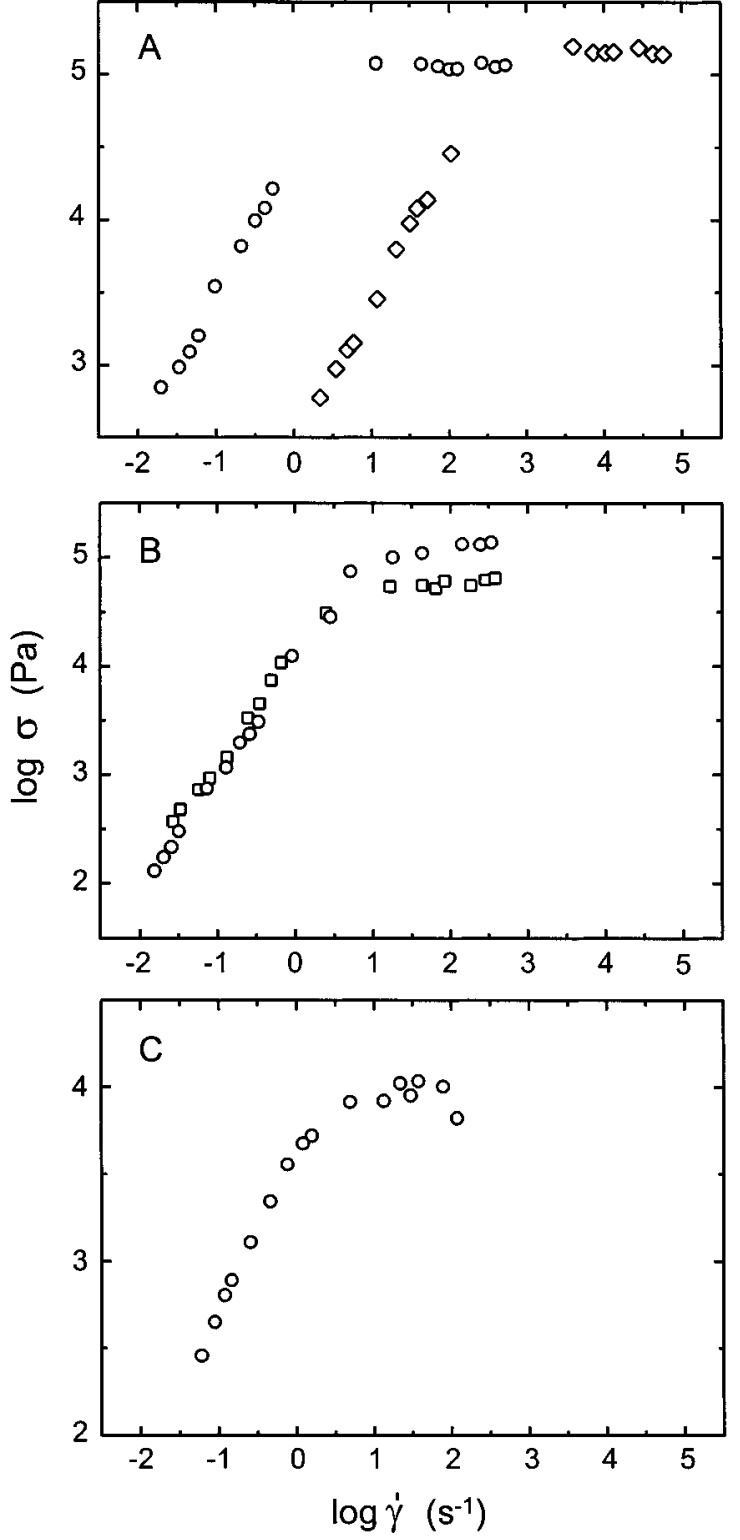

Figure 7. The data for the loss modulus $\left(G^{\prime \prime}{ }_{\text {eff }}\right)$ in Figure 6 are presented here as viscous shear stress $(\sigma)$ vs shear rate (strain multiplied by angular frequency). As in Figure 6, the diamond symbols in panel A show results from an additional experiment at $130 \mathrm{~Hz}$. A limiting value of the shear stress was reached at a strain of $0.1,0.1$, and 0.06 for (A) hydroxyl, DOL; (B) piperonyl, AM2001; and (C) p-phenoxyanilinium salt of carboxylic acid, respectively.

adsorbed polymer chains (polymer brushes in a solvent), ${ }^{55}$ even though such a structure in polymer melts has not been confirmed. It is possible that a much longer equilibration time than expected from the bulk viscosity is needed in this separation range due to the affinity of the functionalized end groups of our samples for the surface (effectively causing an additional, weak linking of the end of the chain to the surface) and that the repulsion is still a nonequilibrium effect. It was unexpected that the interactions in the highly viscous $p$-phenoxyaniliniumterminated sample would be measurable within a similar experimental time as for the other systems.

For the final, seemingly incompressible film obtained at small separations, which is commonly seen as two confined surfaces interact at molecularly thin spacings across a polymer melt, it has similarly been argued that it occurs because the relaxation time needed exceeds the experimental time or because of pinning of the polymer chains to the solid surfaces ${ }^{69}$ Layering in films of linear, perfluorinated polymer confined between two surfaces has been observed in experiments on unfunctionalized Fomblin- $\mathrm{Z}$ of higher molecular weight during shear at large pressures, where the thickness decreased in steps of $8 \AA{ }^{6,7}$ which is close to the estimated molecular segment diameter of approximately $7 \AA .20,31,39,40 \mathrm{We}$ did not observe any decrease in the layer thickness even at the larger pressures studied, but we note that the compressed layer thickness for DIAC, DOL, and AM2001 was 24,33 , and $40 \AA$, respectively, which corresponds closely to integer numbers of molecular segment diameters. The thickness of the compressed $p$-phenoxyanilinium salt film was considerably larger. We also note that experiments ${ }^{47}$ on higher molecular weight hydroxyl- and piperonyl-terminated Fomblin-Z revealed that, for these polymers, larger film thicknesses were obtained for higher molecular weights at a given normal pressure. The absence of an oscillatory interaction force as a function of surface separation (Figure 1) suggests that a semiordered layering (as in thin films of linear alkanes ${ }^{55}$ or poly(dimethylsiloxane) ${ }^{68}$ confined between atomically smooth surfaces) was hindered in our end-functionalized systems, even though it has been demonstrated that the perfluorinated backbone of DIAC,,$^{21}$ DOL, ${ }^{21,39,40}$ and AM2001 ${ }^{30}$ adopts an orientation parallel to polar surfaces. The influence of localization of end groups to the solid surface and of end group-end group interactions on the structure of the confined film will be discussed further below.

Forces Acting upon Retraction of the Solid Surfaces. The adhesion measured upon slow separation of the solid surfaces differed markedly according to the sample. We note again that although the high viscosity of these samples makes it more difficult to measure the equilibrium adhesion, these measurements were possible provided that the time between each movement and the subsequent measurement was sufficiently long. As a result of the long equilibration times, the film had in general been compressed for about $1 \mathrm{~h}$ before the separation occurred. The adhesions observed do not correlate with the bulk viscosities (note especially the particularly low adhesion obtained for the $p$-phenoxyanilinium salt, Figure 1D). Furthermore, the adhesion does not correlate with the film thickness as might be expected if it were due to layering in a confined film. The van der Waals attraction $\left(F / R=-A / 6 D^{2}\right.$, where the Hamaker constant calculated according to van der WaalsLifshitz theory ${ }^{55}$ is $A=2.47 \times 10^{-20} \mathrm{~J}$ for mica interacting across Fomblin- $Z$ with $\epsilon=2.03^{70}$ and $n=1.294^{70}$ ) is very small at the separations where adhesion was observed in these systems and cannot account for the values measured. In a previous study ${ }^{71}$ on rate-dependent adhesion it has been suggested that possible bridging of difunctionalized chains (hydroxyl-terminated Fomblin-Z) from one surface to the other might occur. We will return to the adhesion results after discussing possible structure of the film.

Dynamic Structure of the Confined Films. The lubricating properties of these samples seemed to correlate with their film thickness obtained on maximum compression. We emphasize that there is no obvious correlation between this film thickness and the bulk viscosity of the samples (cf. Table 1). It has been suggested $^{5}$ that a polymer melt of higher viscosity may have more favorable lubricating properties. This can be true for polymers of identical chemical composition but different molecular weight, since the thickness of the immobilized film usually increases with the molecular weight (as does the bulk viscosity), and a thicker lubricating film between the sliding surfaces will in general protect the surfaces better from wear, but the situation is evidently more complicated in the present 
case, where molecules of similar molecular weight differ in their chain-end functionality.

For the oligomers in this study, which were all of a similar chain length, the different viscosity values (Table 1) gave an indication of the different interactions between the polar end groups of the chains in the bulk compared to unfunctionalized chains. The situation is complex since these end group-end group interactions were still present in a thin film and might be expected to compete with the interactions of the end groups with the solid surface.

For evaluation of the effects of end groups, it would be desirable to compare the lubricating properties of our samples directly with experimental data on unfunctionalized Fomblin$Z$. There is currently no unfunctionalized Fomblin- $Z$ commercially available with a molecular weight as low as the samples in this study. The lowest molecular weight available, $M_{\mathrm{n}}=2900 \mathrm{~g} \mathrm{~mol}^{-1}$, has a bulk viscosity of $0.52 \mathrm{P}$ at $25{ }^{\circ} \mathrm{C}$ and was found to form a $30 \AA$ thick film between mica surfaces on confinement under conditions similar to the ones stated above. In low-amplitude shear experiments ${ }^{47}$ on this polymer, we found elastic and viscous moduli around 6 and $2 \mathrm{MPa}$, respectively, and a discontinuous transition from the linear to nonlinear regime as the shear amplitude was increased. Similar results (elastic and viscous moduli around 10 and $2 \mathrm{MPa}$, respectively) have been obtained in a shear study of an unfunctionalized Fomblin sample with a molecular weight around $10000 \mathrm{~g} \mathrm{~mol}^{-1}$ which formed a $25 \AA$ thick film when used as received, without drying over sieves (Y.-K. Cho, unpublished results).

The thin-film rheological properties of DIAC are similar to the results described above for an unfunctionalized sample that is not expected to have any specific interaction with the polar surface (other than possible location of end groups near the surface for entropic reasons ${ }^{72}$ ). It is, however, unlikely that a polar group like carboxylic acid ${ }^{21,42}$ would fail to physisorb to a polar surface as, for example, the hydroxyl groups in DOL, ${ }^{19-26,28,29,32-35,38-40,73}$ the piperonyl groups in AM2001, 30,31 or the $p$-phenoxyanilinium group ${ }^{41}$ are expected to do.

The main reason for the different shear properties of these functionalized samples is thus most likely not due to markedly different affinity of the end groups for the solid surface but has to be sought elsewhere. Since the shear plane is likely within the confined film and not at the interface between the film and the surface, the properties of the whole confined film have to be considered. It is evident that the bulk viscosities for the samples in this study are higher than that for an unfunctionalized sample of corresponding or slightly higher molecular weight ${ }^{14}$ (cf. Table 1 and discussion above). The different viscosities arise from interactions between the polar end groups of the chains and particularly from hydrogen bonding between the end groups in the DIAC ${ }^{74}$ and $\mathrm{DOL}^{21}$ samples.

It has been demonstrated that carboxylic acid terminated Fomblin- $Z$ exists predominantly in the dimerized form in the bulk, ${ }^{74}$ i.e., each carboxylic acid end group forms two hydrogen bonds with another carboxylic acid group. Generally, in a carboxylic acid dimer, the two hydrogen bonds form a planar structure that has very low dipole moment, and no further hydrogen bonding to these two "coupled" groups can occur. ${ }^{75}$ In contrast, the hydroxyl-terminated chains can form only one hydrogen bond between two end groups. The hydrogen atom left on one of the hydroxyl groups can form a hydrogen bond to a third end group, etc. ${ }^{76}$ The resulting temporary structure in the hydroxyl-terminated polymer is thus clusterlike instead of linear (chainlike) as in the carboxylic acid. ${ }^{75,76}$ It is conceivable that the transient structure of the carboxylic acid terminated polymer is similar to the unfunctionalized sample, which only consists of linear chains. It has been shown that several linear polymers, ${ }^{3,4}$ including a high molecular weight Fomblin-Z, 6,7 easily solidify on confinement, as evidenced by the formation of high shear moduli and a large limiting shear stress. Similar results have been obtained in experiments ${ }^{8}$ on a different linear perfluoropolyalkyl ether, Demnum S-20, ${ }^{77}$ with a molecular weight similar to the samples in our study. The structure in the DOL and in the AM2001, where aggregation may occur through polar interactions, can tentatively be compared to results for branched polymer chains, including a high molecular weight Fomblin- $\mathrm{Y}^{6}$ and a Krytox ${ }^{78}$ sample of molecular weight similar to the samples in this study. ${ }^{8}$ The branched polymers are more fluidlike (i.e., less likely to solidify at low pressure) and function as good lubricants. It was interesting to note that the Krytox sample ${ }^{8}$ showed a similar, unusual frequency dependence of the shear modulus as seen for the DOL and $p$-phenoxyaniliniumterminated samples in Figure 2.

On the basis of the solubility of DOL, AM2001, and the $p$-phenoxyanilinium salt in various solvents (cf. Experimental section and ref 35) and their relative ability to dissolve glue (cf. Experimental section), it is reasonable to assume that the polarity of these samples increased in the order DOL $\leq$ AM2001 $\ll p$-phenoxyanilinium salt, which corresponds to the film thickness formed on confinement and the resulting lubricating properties. It is possible that the structure formed as a result of hydrogen bonding and polar interactions between the end groups of the DOL and AM2001 was similar in both films, since these samples have quite similar nanorheological properties, particularly at increased pressure.

On the basis of the arguments presented above, one might expect these perfluorinated molecules to form structures owing to association of the chain end groups; such structures would be analogous to the micellar and block copolymer microstructures that are well-known to form in hydrocarbon systems. We have not found direct evidence in the literature of such selfassociation in the bulk. However, a recent study by Xu et al. ${ }^{29}$ suggests that Fomblin-Z lubricants modified with aromatic end groups have stronger interactions with polar surfaces than hydroxyl-terminated ones and that association in the lubricant film may occur.

Interactions of End Groups with the Solid Surface. The end groups in our study did not form covalent attachments to the solid surface, but polar end groups are expected to be located on average close to the polar mica. Models for the dependence of the shear stress on shear rate have been proposed for cases where a polymer interacts either strongly 9,10 or weakly ${ }^{11}$ with a solid surface. In both cases, the stress is expected to increase linearly with the shear rate at small deformations (linear response regime); this we also observed experimentally. In the case of weak attraction to a confining surface, it has been suggested ${ }^{11}$ that the stress in the nonlinear regime increases as the shear velocity to the power $1 / 3$, while for strong interactions and bridge formation, a plateau in the stress with no dependence on shear velocity or a dependence to the power $-1 / 3$ is followed by a region of stress increasing as velocity to the power $1 / 3 .{ }^{9,10}$ Experimentally, we observed that the stress in the nonlinear regime for the AM2001 and p-phenoxyanilinium-terminated samples increased slowly with shear rate, while an indication of a plateau, followed by a slow increase, was found for DIAC and DOL. At larger pressure a plateau in the shear stress was seen for DOL, whereas the stress continued to increase slowly in AM2001 and the p-phenoxyanilinium salt. 
The presence of molecules bridging the two surfaces has been suggested in a study of rate-dependent adhesion, ${ }^{71}$ where DOL showed a slightly stronger increase in adhesion force with separation rate than was observed for monofunctionalized samples. It is possible that such bridging would contribute to the adhesion observed in Figure 1. The separation could also occur from adhesive minima in a layered structure as has been seen in other polymer systems. It has been shown that, for poly(dimethylsiloxane), the outer minima of oscillatory static forces are very shallow ${ }^{68}$ and if this is the case in our films, such minima might not have been detectable. Layering might possibly explain the adhesion in the DIAC system, especially in view of the "linear chain" structure we suspect for this system, since the film thickness is only about three molecular segment diameters. The adhesion observed for the DOL and AM2001 samples is, however, not explained by such an approach since it was larger even though the compressed layer thicknesses in these systems are larger.

Comparison with Other Characterization Methods. In comparison with experiments performed with instruments strongly resembling the recording/reading mechanism in magnetic storage devices, we find similarities in addition to the affinity of the polar end groups for the surface that was already discussed above. The DOL and AM2001 appear to have more favorable lubricating properties than unfunctionalized Fomblin$\mathrm{Z}$, as has been found previously in different types of sliding experiments. ${ }^{19,23-25,28,29,38}$ On a magnetic tape covered with lubricant and $\mathrm{SiO}_{2}$ particles, ${ }^{27}$ it was found that a system lubricated with Fomblin-Z octadecylamine had a lower friction coefficient than AM, DOL, and DIAC samples. It is, however, not feasible to compare our data directly to the results for these systems in ref 27 , where the sliding occurred in contact with a large number of particles.

The performance of a lubricant is, however, determined not only by its friction coefficient (i.e., by its sensitivity to load or pressure) but also by the absolute magnitudes of the friction force and the stability of the film. Upon comparison of the DOL and AM2001 samples, we find that their behavior was quite similar, with the AM2001 having lower values, possibly correlated with the slightly larger film thickness. For practical applications, their different stability toward temperature and moisture becomes an important consideration. It is known that the end group of AM2001 easily decomposes to form a hydroxyl group. ${ }^{35}$ The $p$-phenoxyanilinium-terminated sample exhibits very favorable lubricating properties, i.e., a relatively low friction force, but the large film thickness (around $100 \AA$ ) formed spontaneously on confining the melt might be a disadvantage, since at the angular velocities reached in a storage device, polymer from a thick film migrates toward the edge of the disk. ${ }^{79,80}$ Furthermore, the large film thickness might be a disadvantage for contact recording where a low film thickness is desirable. It might be possible to form a thinner film than the one obtained here for the compressed melt by the dipping process employed in industry. ${ }^{36}$

\section{Conclusions}

We have measured the tribological properties of thin films of four end-functionalized linear perfluoropolyalkyl ethers (Fomblin-Z) of similar molecular weight and chain composition but different end group functionality, confined between atomically smooth solid surfaces. The shear properties of these polymer films were markedly different for polar end-groups that were expected to have similar, nonspecific affinity for the solid surface. The different results were explained in terms of end group-end group interactions within the interior of the confined films, specifically as different structures formed by hydrogen bonding between carboxylic acid terminated chains compared to hydroxyl- and piperonyl-terminated ones. It is interesting to consider the unusually thick films comprised of the chains terminated by the $p$-phenoxyanilinium salt of a carboxylic acid. Taken together, these results suggest a significant influence of self-association in systems that include polar chain ends in an otherwise perfluorinated environment.

Acknowledgment. We thank Yoon-Kyoung Cho (University of Illinois) for helpful discussions and for making available the data on an unfunctionalized higher molecular weight Fomblin. We also thank Asako Koike (Hitachi Corporation) for helpful discussions. This work was supported by the Hitachi Corporation and by the National Science Foundation.

\section{References and Notes}

(1) Israelachvili, J.; McGuiggan, P. M.; Gee, M.; Homola, A.; Robbins. M.; Thompson, P. J. Phys.: Condens. Matter 1990, 2, SA89.

(2) Van Alsten, J.; Granick, S. Macromolecules 1990, 23, 4856.

(3) Granick, S.; Hu, H.-W. Langmuir 1994, 10, 3857.

(4) Peanasky, J.; Cai, L. L.; Granick, S.; Kessel, C. R. Langmuir 1994, 10,3874 .

(5) Luengo, G.; Schmitt, F.-J.; Hill, R.; Israelachvili, J. N. Macromolecules 1997, 30, 2482.

(6) Homola, A. M.; Nguyen, H. V.; Hadziioannou, G.J. Chem. Phys. 1991, 94, 2346

(7) Hirz, S. J.; Homola, A. M.; Hadziioannou, G.; Frank, C. W. Langmuir 1992, 8, 328.

(8) Cho, Y.-K.; Granick, S. Wear 1996, 200, 346.

(9) Subbotin, A.; Semenov, A.; Mainas, E.; Hadziioannou, G.; ten Brinke, G. Macromolecules 1995, 28, 1511.

(10) Subbotin, A.; Semenov, A.; Hadziioannou, G.; ten Brinke, G. Macromolecules 1996, 29, 1296.

(11) Subbotin, A.; Semenov, A.; Hadziioannou, G.; ten Brinke, G. Macromolecules 1995, 28, 3901.

(12) Hu, H.-W.; Granick, S.; Schweizer, K. S. J. Noncryst. Solids 1994, 172-174, 721 .

(13) Sianesi, D.; Zamboni, V.; Fontanelli, R.; Binaghi, M. Wear 1971, 18,85 .

(14) Marchionni, G.; Ajroldi, G.; Pezzin, G. Eur. Polym. J. 1988, 24, 1211.

(15) Herrera-Fierro, P.; Jones, W. R., Jr.; Pepper, S. V. J. Vac. Sci. Technol. A 1993, 11, 354.

(16) Jones, W. Tribology Trans. 1995, 38, 557.

(17) Gschwender, L.; Snyder, C. E., Jr.; Oleksiuk, M.; Koehler, M. Tribology Trans. 1996, 2, 368.

(18) Ausimont S.p.A., product information

(19) Homola, A. M.; Mate, C. M.; Street, G. B. MRS Bull. 1990, 15, 45 .

(20) Mate, C. M. Phys. Rev. Lett. 1992, 68, 3323.

(21) Yanagisawa, M. Tribology Trans. 1993, 36, 484. Yanagisawa, M. Wear 1993, 168, 167. Yanagisawa, M. Tribology Trans. 1994, 37, 629.

(22) Rühe, J.; Blackman, G.; Novotny, V. J.; Clarke, T.; Street, G. B.; Kuan, S. J. Appl. Polym. Sci. 1994, 53, 825

(23) Rühe, J.; Novotny, V.; Clarke, T.; Street, G. B. J. Tribology 1996, 118,663 .

(24) Koinkar, V. N.; Bhushan, B. J. Appl. Phys. 1996, 79, 8071. Koinkar, V. N.; Bhushan, B. J. Vac. Sci. Technol. A 1996, 14, 2378.

(25) Bhushan, B.; Zhao, Z. IEEE Trans. Magn. 1997, 33, 918.

(26) Perry, S. S.; Mate, C. M.; White, R. L.; Somorjai, G. A. IEEE Trans. Magn. 1996, 32, 115.

(27) Kondo, H.; Hisamichi, Y.; Kamei, T. J. Magn. Magn. Mater. 1996, $155,332$.

(28) Gui, J.; Kuo, D.; Marchon, B.; Rauch, G. C. IEEE Trans. Magn. 1997, 33, 932.

(29) Xu, C.-B.; Frank, C. W.; Tang, W. T.; Terrill, C. J. Adhes. 1998 , 67, 195.

(30) Toney, M. F.; Thompson, C. J. Chem. Phys. 1990, 92, 3781

(31) Gao, C.; Dai, P. IEEE Trans. Magn. 1997, 33, 3118.

(32) Dai, Q.; Vurens, G.; Luna, M.; Salmeron, M. Langmuir 1997, 13, 4401.

(33) Min, B. G.; Choi, J. W.; Brown, H. R.; Yoon, D. Y.; O’Connor, T. M.; Jhon, M. S. Tribology Lett. 1995, 1, 225.

(34) O'Connor, T. M., Back, Y. R.; Jhon, M. S.; Min, B. G.; Yoon, D. Y.; Karis, T. E. J. Appl. Phys. 1996, 79, 5788.

(35) Viswanathan, K. V. J. Appl. Phys. 1994, 75, 6161. 
(36) Gao, C.; Lee, Y. C.; Chao, J.; Russak, M. IEEE Trans. Magn. 1995, $31,2982$.

(37) Hahm, C. D.; Bhushan, B. J. Appl. Phys. 1997, 81, 5384.

(38) Gellman, A. J. Curr. Opin. Colloid Interface Sci. 1998, 3, 368.

(39) Tyndall, G. W.; Leezenberg, P. B.; Waltman, R. J.; Castenada, J. Tribology Lett. 1998, 4, 103.

(40) Waltman, R. J.; Pocker, D. J.; Tyndall, G. W. Tribology Lett. 1998, $4,267$.

(41) Shouji, M.; Imazeki, S.; Usami, K. J. Jpn. Soc. Tribologists 1998 , 43,242 .

(42) Kasai, P. H. J. Appl. Polym. Sci. 1995, 57, 797.

(43) Danusso, F.; Levi, M.; Gianotti, G.; Turri, S. Eur. Polym. J. 1994 30,1449 .

(44) Tieghi, G.; Levi, M.; Imperial, R. Polymer 1998, 39, 1015.

(45) Fomblin is a registered trademark of Ausimont S.p.A.

(46) Sanguineti, A.; Gurada, P. A.; Marchionni, G.; Ajroldi, G. Polymer 1995, 36, 3697.

(47) Ruths, M.; Granick. S. Tribology Lett., submitted.

(48) Wallington, T. J.; Schneider, W. F.; Sehested, J.; Bilde, M.; Platz, J.; Nielsen, O. J.; Christensen, L. K.; Molina, M. J.; Molina, L. T.; Wooldridge, P. W. J. Phys. Chem. A 1997, 101, 8264.

(49) Israelachvili, J. N.; Adams, G. E. J. Chem. Soc., Faraday Trans. 1 1978, 74, 975

(50) Van Alsten, J.; Granick, S. Phys. Rev. Lett. 1988, 61, 2570.

(51) Peachey, J.; Van Alsten, J.; Granick, S. Rev. Sci. Instrum. 1991, $62,463$.

(52) Israelachvili, J. N. J. Colloid Interface Sci. 1973, 44, 259.

(53) Watanabe, H.; Matsuyama, S.; Mizutani, Y.; Kotaka, T. Macromolecules 1995, 28, 6454.

(54) Horn, R. G.; Hirz, S. J.; Hadziioannou, G.; Frank, C. W.; Catala, J. M. J. Chem. Phys. 1989, 90, 6767.

(55) Israelachvili, J. N. Intermolecular and Surface Forces, 2nd ed.; Academic Press: London, 1991.

(56) Granick, S.; Demirel, A. L.; Cai, L. L.; Peanasky, J. Isr. J. Chem. 1995, 35,75 .
(57) Hertz, H. J. Reine Angew. Math. 1881, 92, 156

(58) Johnson, K. L.; Kendall, K.; Roberts, A. D. Proc. R. Soc. London, Ser. A 1971, 324, 301 .

(59) McLeish, T. C. B. J. Polym. Sci., Polym. Phys. 1987, 25, 2253.

(60) Cates, M. E. J. Phys.: Condens. Matter 1996, 8, 9167.

(61) Grand, C.; Arrault, J.; Cates, M. E. J. Phys. II 1997, 7, 1071.

(62) Sollich, P.; Lequeux, F.; Hebraud, P.; Cates, M. E. Phys. Rev. Lett.

1997, 78, 2020.

(63) Soltero, J. F. A.; Bautista, F.; Puig, J. E. Manero, O. Langmuir 1999, 15, 1604

(64) Johnson, K. L.; Tevaarwerk, J. L. Proc. R. Soc. A 1977, 356, 215.

(65) Högland, E.; Jacobson, B. O. J. Tribology 1986, 108, 571.

(66) Evans, C. R.; Johnson, K. L. Proc. Inst. Mech. Eng. 1986, 200, 303.

(67) Bair, S.; Winer, W. O. J. Tribology 1992, 114, 1.

(68) Horn, R. G.; Israelachvili, J. N. Macromolecules 1988, 21, 2836.

(69) de Gennes, P.-G. C. R. Acad. Sci., Ser. II (Paris) 1987, 305, 1181.

(70) Marchionni, G.; Ajroldi, G.; Pezzin, G. Rheology and Tribology of Engine Oils; Society of Automotive Engineers: Warrendale, PA, 1992; p 87.

(71) Ruths, M.; Granick, S. J. Phys. Chem. B 1998, 102, 6056.

(72) Pakula, T. J. Chem. Phys. 1991, 95, 4685.

(73) Paserba, K.; Shukla, N.; Gellman, A. J.; Gui, J.; Marchon, B. Langmuir 1999, 15, 1709.

(74) Doan, V.; Köppe, R.; Kasai, P. H. J. Am. Chem. Soc. 1997, 119 9810

(75) Pimentel, G. C.; McClellan, A. L. The Hydrogen Bond; Freeman: San Francisco, 1960; p 16.

(76) Pimentel, G. C.; McClellan, A. L. The Hydrogen Bond; Freeman: San Francisco, 1960; p 19

(77) Demnum is a registered trademark of Daikin Industries.

(78) Krytox is a registered trademark of E. I. du Pont de Nemours and Company.

(79) Yanagisawa, M. J. Appl. Phys. 1987, 61, 1034.

(80) Middleman, S. J. Appl. Phys. 1987, 62, 2530. 